\title{
Refractory Pancreatic Carcinoma
}

National Cancer Institute

\section{Source}

National Cancer Institute. Refractory Pancreatic Carcinoma. NCI Thesaurus. Code C142808.

Pancreatic carcinoma that does not respond to treatment. 